\title{
Bronchoscopic Long-Term Palliation of a Recurrent Atypical Carcinoid Tumor
}

\author{
Stamatis Katsenos Jose Rojas-Solano Maren Schuhmann Heinrich D. Becker \\ Department of Interdisciplinary Endoscopy, Thoraxklinik, Heidelberg University, Heidelberg, Germany
}

For editorial comment see p. 285

\section{Established Facts}

- Surgery is the treatment of choice for bronchial carcinoid tumors.

\section{Novel Insights}

- In case of inoperability, endoscopic treatment might be an alternative for long-term palliation even in recurrent disease.

\section{Key Words}

Bronchial atypical carcinoid • Bronchoscopic treatment • Nd:YAG laser

\begin{abstract}
Bronchial carcinoid tumors account for $1-2 \%$ of all primary lung tumors and are separated into 2 subgroups: typical and atypical carcinoids. Atypical carcinoids as intermediategrade malignancies can metastasize more frequently, thus exhibiting poorer prognosis than the low-grade typical carcinoid tumors. Surgical resection remains the mainstay of treatment for pulmonary carcinoids. Bronchoscopic treatment using ablation techniques is an effective alternative to surgery in selected patients with typical carcinoid tumors. However, evidence is lacking regarding the effect of bronchoscopic resection of atypical carcinoid tumor and its recurrences. We report the case of a 73-year-old male with fre-
\end{abstract}

quent endobronchial recurrences of a previously surgically resected atypical carcinoid tumor successfully treated using $\mathrm{Nd}$ :YAG laser photoresection. Furthermore, the therapeutic and local staging aspects of the disease are discussed emphasizing the efficacy of bronchoscopic resection strategies and the value of novel bronchoscopic imaging techniques in detailed inspection of the structures of the bronchial wall.

Copyright $\odot 2011$ S. Karger AG, Basel

\section{Introduction}

Bronchial carcinoids represent $<2 \%$ of all primary lung malignancies and are divided into 2 subtypes: typical and atypical carcinoid tumors according to the recent histological classification of lung tumors by the World Health Organization [1]. This distinction of bronchial carcinoids is clinically significant as typical carcinoids

\section{KARGER}

Fax +4161306 1234

E-Mail karger@karger.ch

www.karger.com (c) $2011 \mathrm{~S}$. Karger AG, Basel

$0025-7931 / 11 / 0814-0345 \$ 38.00 / 0$

Accessible online at:

www.karger.com/res
Heinrich D. Becker, MD, FCCP

Thoraxklinik at Heidelberg University, Department of Interdisciplinary Endoscopy Amalienstrasse 5

DE-69126 Heidelberg (Germany)

Tel. +496221 396 1251, Fax +49 6221396 246, E-Mail hdb@ @ronchology.org 
Fig. 1. Bronchoscopy showing polypoid lesions in the lower trachea (a), main carina (b) and in the right lower lobe (c) (black arrows).
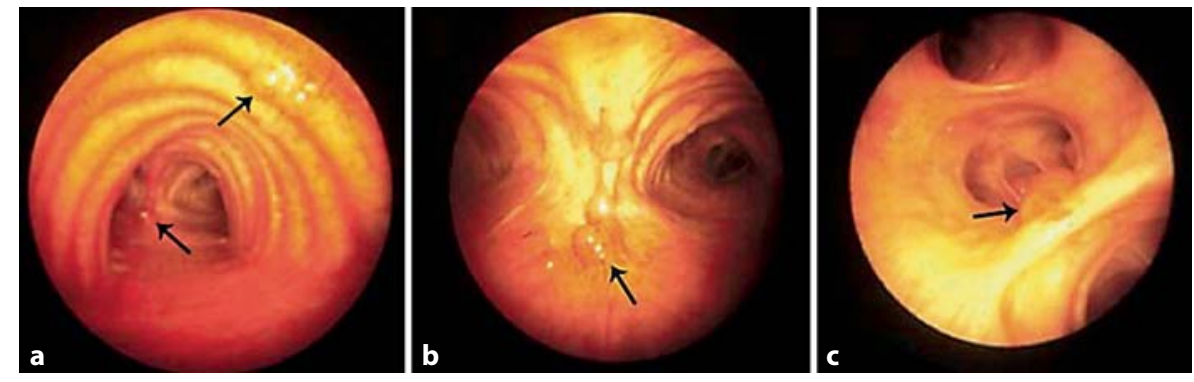

have an excellent prognosis with a 10-year survival rate of $90 \%$ whereas atypical carcinoids have a higher rate of metastases with a 10 -year survival rate of $<60 \%[2,3]$.

Complete surgical resection remains the mainstay of treatment for bronchial carcinoids $[4,5]$. However, bronchoscopic resection using a variety of modalities has turned out to be an efficient alternative to surgical treatment in a selected subset of patients with typical strictly intraluminal bronchial carcinoids [6, 7]. Furthermore, there is a lack of data regarding the usefulness of intraluminal bronchoscopic techniques in the treatment of atypical carcinoid and particularly local recurrences.

In this paper, we describe a patient with previous surgically extirpated bronchial atypical carcinoid, presenting with several local relapses throughout his long-term follow-up that were satisfactorily treated by means of endobronchial Nd:YAG (neodymium-yttrium aluminum garnet) laser.

\section{Case Report}

The patient's history dates back 25 years to 1984, when he had undergone surgical 'enucleation' of an intraluminal polypoid mass obstructing the medial segment of the right middle lobe. Histopathology was described as atypical carcinoid tumor. After that, he underwent regular clinical and radiological follow-up from 1985 to 1991, which revealed a well-defined rounded opacity $<1 \mathrm{~cm}$ in diameter that was located in the right lower lobe. On serial chest radiographs there was no growth.

Nine years after surgery, in 1993, the patient was referred to our department for further evaluation since the lesion had increased in size as seen by chest X-ray and computed tomography (CT). The patient was asymptomatic at presentation. Fiberoptic bronchoscopy showed polypoid lesions occluding the segments of the right lower lobe and lesions in the lower trachea and main carina (fig. 1a-c). Biopsies confirmed lesions of an atypical carcinoid tumor. Laboratory testing, including serotonin and urinary 5-hydroxyindoleacetic acid levels, were normal. Further imaging including abdominal CT did not reveal any metastatic deposit while somatostatin receptor scintigraphy showed an in- creased uptake in the right lung without further extrathoracic foci.

The strategy regarding therapeutic options for our patient was the following: (1) surgical management of the pulmonary atypical carcinoid tumor, (2) bronchoscopic Nd-YAG laser destruction of the endobronchial lesions, and (3) if the intrapulmonary lesion would be negative for atypical carcinoid tumor, additional highdose-rate endoluminal brachytherapy would be feasible.

A radical right lower lobectomy in combination with ipsilateral pleurectomy and resection of the medial segment of the right middle lobe bronchus as well as complete mediastinal lymph node dissection were performed. The histopathological examination revealed an atypical carcinoid tumor invading the underlying parietal pleura; no mediastinal lymph node involvement was detected. As the situation was regarded highly palliative, no highdose-rate brachytherapy was performed. The postoperative course was complicated by bronchopleural fistula and consecutive empyema, treated by surgical means. Follow-up consisted of bronchoscopy, chest radiography, CT and, in particular, followup bronchoscopies at 3-month intervals during the first year, on a semi-annual basis during the second year and subsequently once a year.

Six months following surgical resection, in June 1994, a polypoid lesion in the distal trachea was detected by bronchoscopy. Biopsies again showed an atypical carcinoid. After complete endoscopic mechanical resection with the rigid forceps, the patient received locoregional mediastinal radiotherapy using a total dose of $50 \mathrm{~Gy}$ over 5 weeks, resulting in endoscopic/histological complete remission for 7 consecutive years.

After 7 years, in 2001, by routine fiberoptic follow-up bronchoscopy, we found another recurrence in the proximal left main bronchus. The patient underwent bronchoscopic resection by Nd:YAG laser and argon plasma coagulation, as no other lesions were visible (fig. $2 \mathrm{a}-\mathrm{c}$ ).

Four years later, in 2005, a hypodense solitary lesion on the 6th segment of the right liver lobe was noted on abdominal ultrasound. Further investigation with contrast-enhanced CT and positron emission tomography were compatible with metastasis from the atypical carcinoid tumor. Liver segmentectomy was performed, resulting in complete remission since. Also bronchoscopy, including autofluorescence, narrow band imaging, endobronchial ultrasound and biopsies, is negative for tumor recurrence up to now (fig. 2d).

To summarize, our patient has experienced local endobronchial recurrences of an atypical carcinoid tumor 3 times, includ- 
ing 2 intrapulmonary lesions and 1 episode of a distant metastasis. Therapy by surgery and especially bronchoscopic treatment by Nd:YAG laser resulted in long-term palliation for 25 years after the first diagnosis. The patient still undergoes a regular follow-up regimen including annual imaging (chest-abdominal CT, positron emission tomography scan) and bronchoscopy (autofluorescence bronchoscopy and, endobronchial ultrasound, EBUS) for detection of intrapulmonary and extrathoracic metastases and endobronchial lesions.

Figure 3 summarizes the main features of the patient's history.

\section{Discussion}

Bronchial carcinoid tumors are uncommon malignant neuroendocrine neoplasms, comprising 1-2\% of all primary lung tumors [1]. Carcinoids are classified as typical and atypical carcinoids according to the histologic criteria for bronchial carcinoids proposed by Beasley et al. [1] and Travis et al. [2] and currently adopted by the 2004 World Health Organization classification of lung tumors. The typical subtype is considered a low-grade malignancy, while the atypical type appears to be an intermediate-grade malignant tumor. Strictly speaking, typical carcinoids tend to be less aggressive as they are slow growing (low mitotic rate: $<2$ mitoses per $2-\mathrm{mm}^{2}$ area of viable tumor without necrosis), thus exhibiting excellent prognosis with a 10-year survival rate of approximately $90 \%$ [3]. Compared with typical carcinoids, atypical carcinoids present with a higher rate of local metastatic spread and recurrences (mitotic count: 2-10 mitoses per $2-\mathrm{mm}^{2}$ area of viable tumor) resulting in a significantly reduced 10 -year survival rate (approximately $60 \%)[3,8,9]$.

Surgery is the treatment of choice for bronchial carcinoid tumors providing an essentially curative outcome $[4,5]$. The prevailing trend of surgical management for both typical and atypical carcinoids is conservative resection and complete removal of neoplastic tissue with preservation of as much functional lung tissue as possible [10]. However, some authors advocate wider and more aggressive resections in the presence of atypical carcinoids [11]. As a general rule, more extensive resections are mandatory when the condition is complicated by destruction of distal lung parenchyma. Furthermore, systematic lymph node dissection in addition to surgical treatment is increasingly applied as a therapeutic approach of both carcinoids with positive impact on patients' survival $[12,13]$.

Although from an oncologic standpoint, surgery is considered the gold standard in treatment of bronchial
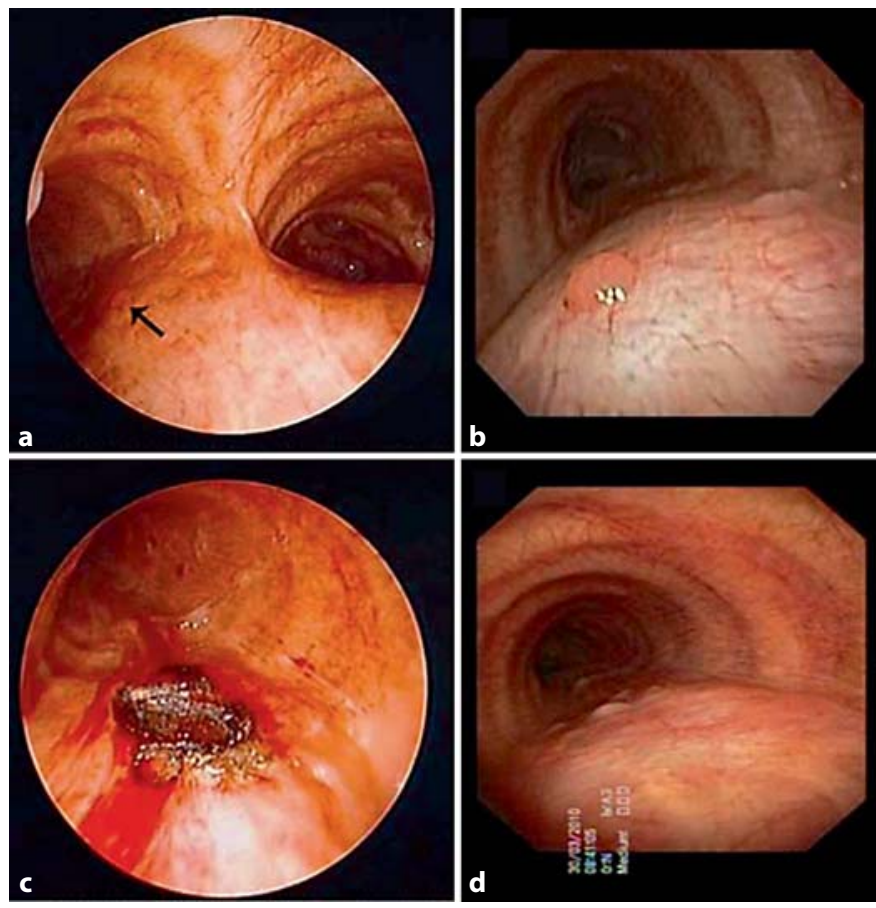

Fig. 2. Bronchoscopy demonstrating a polypoid lesion in the proximal left main bronchus (arrow) (a, b). The lesion was successfully resected by Nd:YAG laser (c). No endobronchial lesion was detected 9 years after the last episode of local recurrence (d).

carcinoids; initial bronchoscopic treatment in special situations may also be an effective 'tissue-sparing' alternative that obviates the need for surgical procedures thus improving patients' quality of life. A variety of bronchoscopic resection techniques have been applied with curative intent, exclusively in strictly intraluminal typical carcinoids that were centrally located, either sessile or presenting with a narrow stalk. However, there are only few published studies addressing this issue $[6,7,14-18]$.

Bronchoscopic treatment was carried out using Nd:YAG laser in most of these studies. Van Boxem et al. [6] reported on 19 patients with resectable endoluminal typical bronchial carcinoid who had undergone bronchoscopic therapy using Nd:YAG laser, photodynamic therapy or brachytherapy under general anesthesia. The tumor was completely eradicated in 14 out of 19 patients (73\%). In the remaining 5 patients, radical surgical resection was performed subsequently, which confirmed the presence of residual atypical carcinoid, in contrast to the initial diagnosis. Cavaliere et al. [7] described their experience with Nd:YAG laser application in patients with resectable typical carcinoids. They recruited 38 patients out 


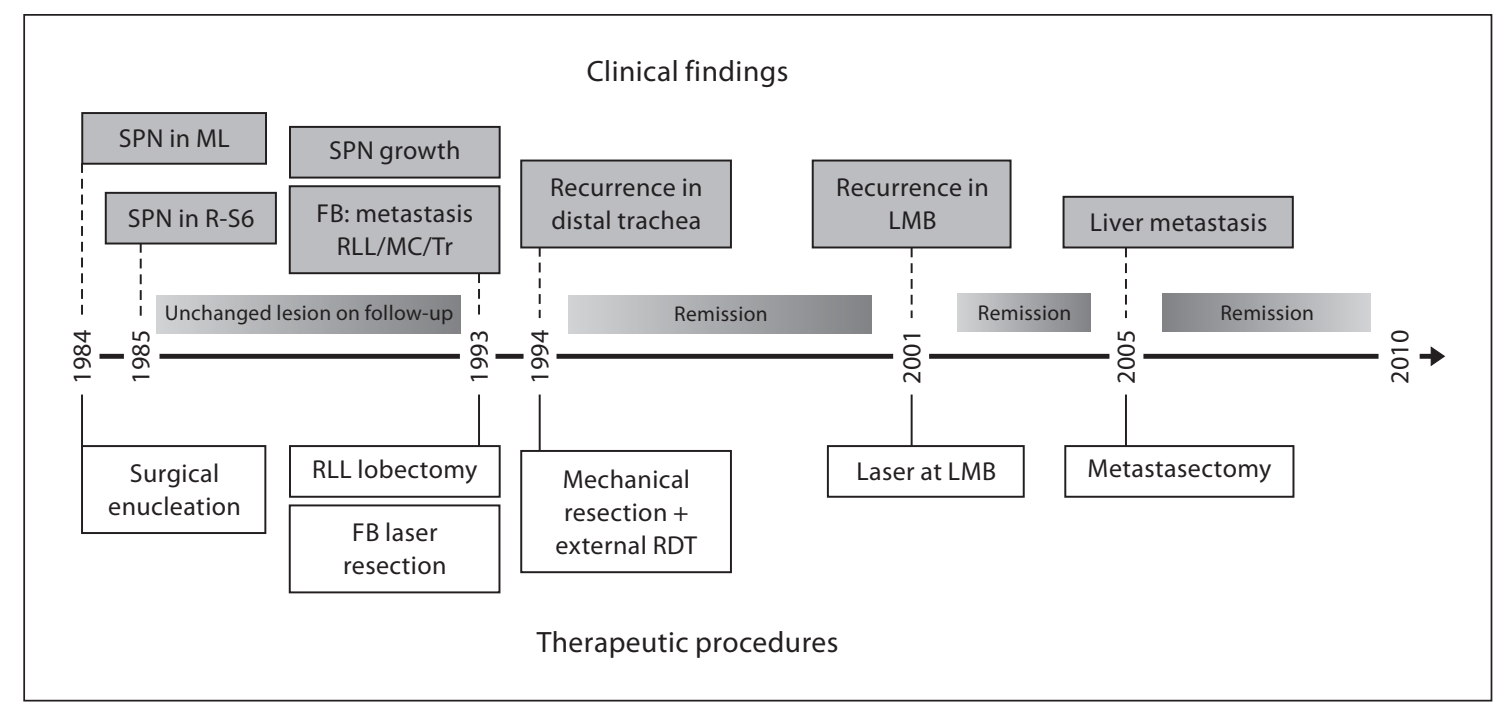

Fig. 3. Patient's history. SPN = Solitary pulmonary nodule; $\mathrm{ML}=$ middle lobe; $\mathrm{R}-\mathrm{S} 6=$ right segment $6 ; \mathrm{FB}=\mathrm{fi}$ beroptic bronchoscopy; RLL = right lower lobe; $\mathrm{MC}=$ main carina; $\mathrm{Tr}=$ trachea; $\mathrm{RDT}=$ radiotherapy; $\mathrm{LMB}=$ left main bronchus.

of 150 (25\%) with typical carcinoids in whom the tumor was entirely intraluminal without evidence of lymph nodal metastases and with a limited base. The success rate was $92 \%$ with a median follow-up of 24 months.

In a recent study by Brokx et al. [14], initial bronchoscopic treatment with Nd:YAG laser or electrocautery and mechanical removal was applied in 72 patients with intraluminal bronchial carcinoids aiming at improving the presurgical condition, obtaining adequate tissue samples for appropriate histologic classification and performing less extensive parenchymal resection. Successful tumor eradication was achieved in 33 of 72 (45\%) cases with a median follow-up of 65 months. One more recent study showed encouraging long-term results in treating patients with typical carcinoids by using mechanical resection through a rigid bronchoscope under general anesthesia. Complete response was observed in 26 out of 28 patients (94\%) with a median observation period of 105 months [15].

Bertoletti et al. [16] reported their experience on a series of 18 patients with intraluminal typical carcinoids, treated bronchoscopically by cryotherapy. Median follow-up was 55 months and a single local recurrence (5.5\%) was noted 7 years after treatment requiring surgery. Fuks and coworkers [17] evaluated the effectiveness of endobronchial laser photoresection in 10 patients with strictly intraluminal typical carcinoids using flexible bronchoscopy under conscious sedation. All patients had complete response, and their median follow-up period was 29 months. In a descriptive study, argon plasma coagulation was successfully used for the treatment of a recurrent intraluminal bronchial typical carcinoid [18].

There are some prerequisites for a curative intent of endobronchial typical carcinoid tumor resection with Nd:YAG laser, according to the above-mentioned studies. The tumor should be strictly intraluminal, located within large airways without signs of peribronchial extension (bronchial wall involvement, nodal metastases) as based on CT scan and EBUS. Moreover, the tumor base should be limited and within the range of the laser. Treatment of the tumor base is of fundamental importance as the risk of local recurrences decreases considerably. However, several authors argue that laser bronchoscopic treatment should be reserved for patients who are medically unfit for surgery or as an adjunct to surgical treatment because it can help to remove tumor-related postobstructive debris and disclose the distal tumor margin enabling the surgeon to perform the most appropriate procedure $[10,19]$.

Initial bronchoscopic treatment using interventional modalities has been effectively used in a series of patients experiencing endobronchial metastases from primary tumors of extrathoracic orgin (e.g. colorectal cancers) [20]. Nevertheless, bronchoscopic laser resection has not been adequately evaluated as the prime therapeutic option of bronchial atypical carcinoids and their local re- 
currences. Surgical management is still considered the most established approach. There is only 1 recent study by Brokx et al. [14] who performed initial bronchoscopic laser resection in 4 patients with intraluminal atypical carcinoid tumors. Complete tumor eradication was achieved in 3 cases not requiring further surgical treatment as there was no sign of residual disease (by videobronchoscopy, biopsy, high-resolution CT images and radial endobronchial ultrasonography). The other patient had metastatic disease at referral and received palliative bronchoscopic treatment. The median follow-up of these cases was 14 months (range 14-74.5). This study underpins that assessment of bronchial carcinoids, intraluminal or extraluminal, using novel imaging modalities (thin-slice high-resolution CT, endobronchial ultrasonography) might be of more clinical significance than histologic classification between the typical and atypical pattern concerning their successful eradication with initial bronchoscopic treatment. In addition, long-term data of this study show that initial bronchoscopic treatment is a potentially tissue-sparing alternative to surgery without adversely affecting its outcome.

In the present case, local recurrences were treated by bronchoscopic laser treatment yielding long-term palliation because the patient is still alive (follow-up 25 years). Radial EBUS is an ideal tool for evaluating the airway wall and associated tumor pathology [21]. It is capable of accurately determining the depth and extent of tumor invasion within the different layers of the bronchial wall, thus influencing the curative endobronchial treatment of malignancies [22]. According to EBUS findings, carcinoid tumor recurrence was considered intracartilaginous, and our patient underwent mechanical tumor removal accompanied by Nd:YAG laser application achieving excellent local control. The role of autofluorescence bronchoscopy is equally important for the confirmation of the extent of tumor in centrally located lung cancers [23]. Herth et al. [24] showed that the addition of EBUS in the assessment of small autofluorescence positive lesions increased specificity in terms of predicting malignancy. This combination strategy has been adopted in our patient's postbronchoscopic treatment surveillance as it provides an accurate tool for the screening of submucosal disease.

In conclusion, this case describes a patient with a formerly resected atypical intrapulmonary carcinoid tumor experiencing frequent endobronchial recurrences that were effectively treated by bronchoscopic laser application. This approach is a valuable tissue-sparing alternative to surgical resection thus avoiding unwarranted thoracotomies and providing quality of life. It should be considered in the presence of a strictly intraluminal carcinoid tumor as established by newer endobronchial diagnostic multimodalities irrespective of its histologic classification. Finally, we suggest that after resection of atypical carcinoid, the patients should be kept under long-term follow-up.

\section{References}

1 Beasley MB, Brambilla E, Travis WD: The 2004 World Health Organization classification of lung tumors. Semin Roentgenol 2005; 40:90-97.

-2 Travis WD, Rush W, Flieder DB, et al: Survival analysis of 200 pulmonary neuroendocrine tumors with clarification of criteria for atypical carcinoid and its separation from typical carcinoid. Am J Surg Pathol 1998;22: 934-944.

$>3$ Mezzetti M, Raveglia F, Panigalli T, et al: Assessment of outcomes in typical and atypical carcinoids to latest WHO classification. Ann Thorac Surg 2003;76:1838-1842.

4 Filosso PL, Rena O, Donati G, et al: Bronchial carcinoid tumors: surgical management and long-term outcome. J Thorac Cardiovasc Surg 2002;123:303-309.

$>5$ Stamatis G, Freitag L, Greschuchna D: Limited and radical resection for tracheal and bronchopulmonary carcinoid tumour. Report on 227 cases. Eur J Cardiothorac Surg 1990;4:527-532.

Bronchoscopic Long-Term Palliation of a Recurrent Atypical Carcinoid
6 van Boxem TJ, Venmans BJ, van Mourik JC, Postmus PE, Sutedja TG: Bronchoscopic treatment of intraluminal typical carcinoid: a pilot study. J Thorac Cardiovasc Surg 1998; 116:402-406.

7 Cavaliere S, Foccoli P, Toninelli C: Curative bronchoscopic laser therapy for surgically resectable tracheobronchial tumors: personal experience. J Bronchol Intervent Pulmonol 2002;9:90-95.

8 Beasley MB, Thunnissen FB, Brambilla E, Hasleton P, Steele R, Hammar SP, et al: Pulmonary atypical carcinoid: predictors of survival in 106 cases. Hum Pathol 2000;31: 1255-1265.

-9 Cardillo G, Sera F, Di Martino M, Graziano P, Giunti R, Carbone L, et al: Bronchial carcinoid tumors: nodal status and long-term survival after resection. Ann Thorac Surg 2004;77:1781-1785.
10 El Jamal M, Nicholson AG, Goldstraw P: The feasibility of conservative resection for carcinoid tumours: is pneumonectomy ever necessary for uncomplicated cases? Eur J Cardiothorac Surg 2000;18:301-306.

11 Marty-Ane CH, Costes V, Pujol TL, Alauzen M, Baldet P, Mary H: Carcinoid tumors of the lung: do atypical features require aggressive management? Ann Thorac Surg 1995;59: 78-83.

12 Garcia-Yuste M, Matilla JM, Cueto A, et al: Typical and atypical carcinoid tumours: analysis of the experience of the Spanish multicentric study of neuroendocrine tumours of the lung. Eur J Cardiothorac Surg 2007;31:192-197.

13 Wurzt A, Benhamed L, Conti M, Bouchindhomme B, Porte H: Results of systematic nodal dissection in typical and atypical carcinoid tumors of the lung. J Thorac Oncol 2009;4:388-394. 
14 Brokx HA, Risse EK, Paul MA, et al: Initial bronchoscopic treatment for patients with intraluminal bronchial carcinoids. J Thorac Cardiovasc Surg 2007;133:973-978.

-15 Luckraz H, Amer K, Thomas L, Gibbs A, Butchart EG: Long-term outcome of bronchoscopically resected endobronchial typical carcinoid tumors. J Thorac Cardiovasc Surg 2006;132:113-115.

16 Bertoletti L, Elleuch R, Kaczmarek D, JeanFrannois R, Vergnon JM: Bronchoscopic cryotherapy treatment of isolated endoluminal typical carcinoid tumor. Chest 2006;130: 1405-1411.
17 Fuks L, Fruchter O, Amital A, Fox BD, Abdel Rahman N, Kramer MR: Long-term followup of flexible bronchoscopic treatment for bronchial carcinoids with curative intent. Diagn Ther Endosc 2009;2009:782961.

18 Orino K, Kawai H, Ogawa J: Bronchoscopic treatment with argon plasma coagulation for recurrent typical carcinoids: report of a case. Anticancer Res 2004;24:4073-4078.

19 Morandi U, Casali C, Rossi G: Bronchial typical carcinoid tumors. Semin Thorac Cardiovasc Surg 2006;18:191-198.

20 Fournel C, Bertoletti L, Nguyen B, Vergnon JM: Endobronchial metastases from colorectal cancers: natural history and role of interventional bronchoscopy. Respiration 2009; 77:63-69.
1 Kurimoto N, Murayama M, Yoshioka S, Nishisaka T, Inai K, Dohi K: Assessment of usefulness of endobronchial ultrasonography in determination of depth of tracheobronchial tumor invasion. Chest 1999;115: 1500-1506.

22 Herth FJF, Becker HD, LoCicero J 3rd, Ernst A: Endobronchial ultrasound in therapeutic bronchoscopy. Eur Respir J 2002;20:118-121.

23 Yasufuku K: Early diagnosis of lung cancer. Clin Chest Med 2010;31:39-47.

24 Herth FJF, Becker HD, LoCicero J 3rd, Ernst A: Endobronchial ultrasound improves classification of suspicious lesions detected by autofluorescence bronchoscopy. J Bronchol Intervent Pulmonol 2003;10:249-252. 